\title{
Final Progress Report: \\ Tools for the Development of \\ High Performance Energy Applications and Systems
}

\author{
August 2006- January 2010 \\ DE-FG02-93ER25176
}

\author{
Barton P. Miller \\ bart@cs.wisc.edu \\ Computer Sciences Department \\ University of Wisconsin \\ 1210 W. Dayton St. \\ Madison, WI 53706-1685
}

\section{Technical Areas of Progress}

This report describes progress made over the course of funding for this project.

Our research includes work on infrastructure for extreme scale tools and applications, and techniques for binary code analysis, instrumentation, and control.

\subsection{Tools and Middleware at Scale}

Our scalability research is based on our MRNet multicast-reduction infrastructure. We have produced tools that operate on real codes and systems at extreme scale, and have enabled others to do the same. In addition, we have produced a new, highly efficient, fail-forward recover technique.

\subsubsection{Infrastructure for Extreme Scale Tools}

Large scale systems are rapidly increasing in size and systems with millions of cores are here. We are faced with the challenge of ensuring system software and tools scale to the level required by these environments. In particular, we require scalable debugging and performance tools as users will need mechanisms to ensure the correctness of their codes and the efficient utilization of the underlying, often unique and complex, target architectures.

More recently, several projects have begun to address this problem by integrating hierarchical communication structures with online aggregation mechanisms, like MRNet or Supermon, into their tools. On the debugger side, HP's Ladebug relies on a tree of debug daemons to control large numbers of tasks and STAT (our own Stack Trace Analysis Tool) [4,7,9] uses MRNet to collect and merge stack traces from an entire parallel application. In the area of performance tools both TAU/Paraprof [1,5] and Open|SpeedShop have demonstrated tools with MRNet.

Using a hierarchical approach is a necessary aspect of a scalable tool design and has proven sufficient at modest system sizes of a few thousand of processors. However, when applied to today's largest systems of 100,000 processors and more, we find that new bottlenecks arise and scalable tool designs require further refinements in order to achieve the necessary scalability. We anticipate that these bottlenecks will prove too great for even the most scalable of current tool designs to sup- 


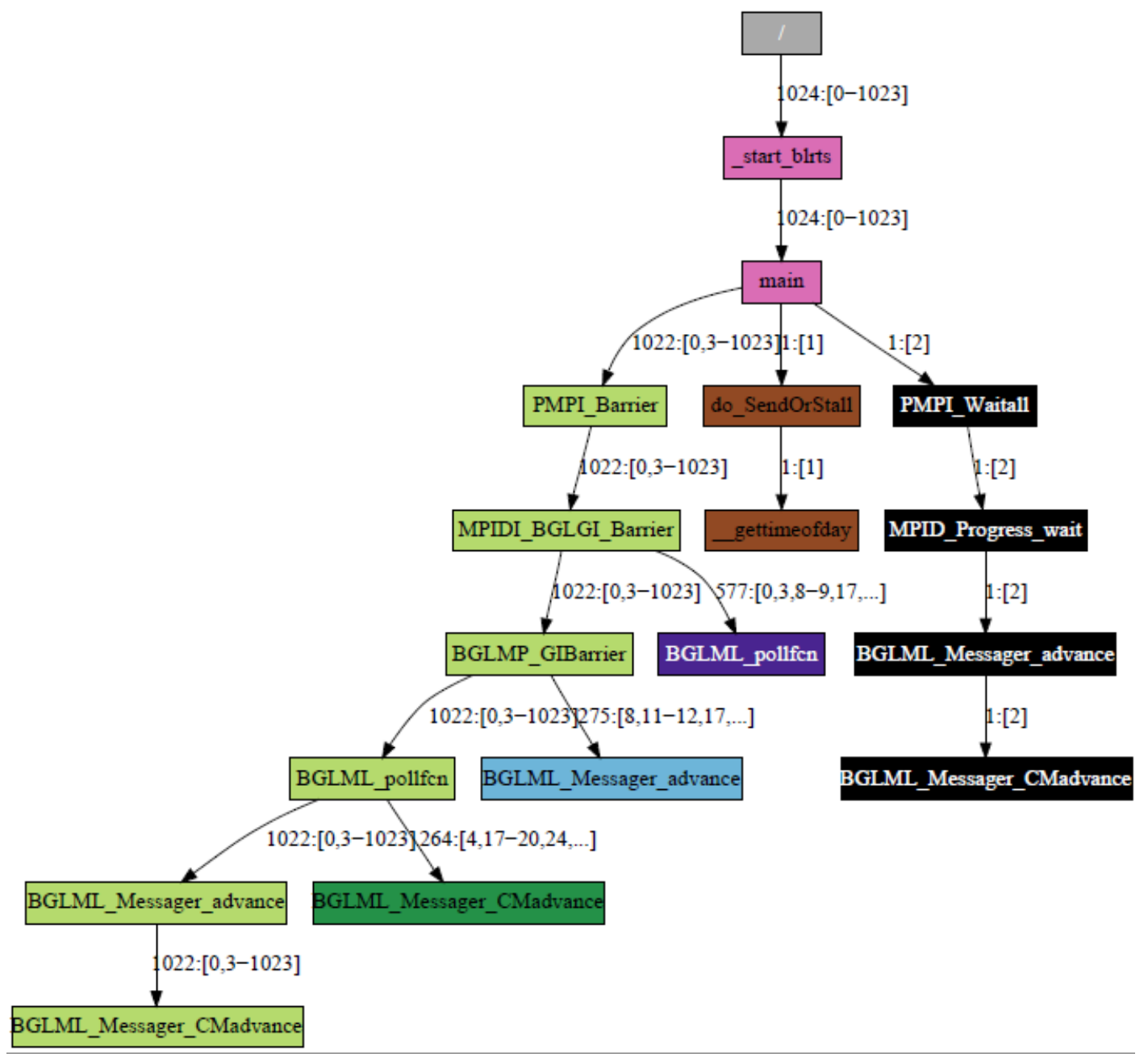

Figure 1: STAT Display for Application with More Than 100,000 Processes

port petascale systems, which are projected to have more than one million cores. Instead, we must enhance those designs with new techniques gleaned from lessons learned on today's largest systems.

The Stack Trace Analysis Tool (STAT) is part of a scalable debugging strategy that uses lightweight tools on an entire parallel application to reduce the problem search space to a manageable subset of tasks, on which a heavyweight debugger can perform root cause analysis. STAT identifies process equivalence classes (groups of processes that exhibit similar behavior) by sampling stack traces over time from each task of the parallel application and merging them into a call graph prefix tree. The call graph prefix tree intuitively represents the application's behavior classes over space and time, as shown in Figure 1. These equivalence classes reduce both the search space and the number of tasks that need to be considered, so that the user can effectively apply a full-featured debugger to problems that arise at scale.

STAT was designed with scalability as the primary goal, so it uses lightweight mechanisms for gathering stack traces and the MRNet [8] tree based overlay network (TBON) to reduce the data and processing loads on its front end. Conceptually, STAT has three main components: the front end, the tool daemons, and the stack trace analysis routine. The front end controls the collection of 
stack trace samples by the tool daemons, and our stack trace analysis routine processes the collected traces. The TBON provides scalable communication between the STAT front end and back ends, while a custom STAT filter efficiently merges the stack traces as they propagate up the communication tree.

The development and deployment of STAT has been quite successful. Our early published results demonstrate that the basic architecture and intelligent implementation of the filter routines support scalability to four thousand tasks. Subsequent experiments have shown that it is the first parallel debugging tool to scale to hundreds of thousands processors while still maintaining reasonable bounds on execution time that allow for interactive usage. Most other tools fail to work at such scales. Some fail due to internal or operating system restrictions. For others, the execution time of even simple, individual operations grows linearly with the scale of the target application, which leads to impractical delays at the scales required for petascale architectures.

Even with an explicitly scalable design such as STAT's, our analysis shows that severe bottlenecks and challenges still remain for petascale tools. In particular, new bottlenecks arise or become more significant at 100,000 or even $1,000,000$ cores compared to 1,000 or 10,000 . While our experiences with STAT may not exhaustively identify all petascale tool performance challenges, most tools will benefit from addressing those we have encountered.

We classify the performance challenges for petascale tools into two main groups: structural problems within the tool and inefficient interactions with the environment. Examples of tool design issues include inefficient analysis algorithms, excessive communication bandwidth requirements, and data structures that use more space than necessary when considered in the aggregate. One can directly address these problems through tool (re)design, e.g., by using different analysis algorithms or data representations. Although STAT's original design handled many of these issues, our experience, consistent with that of scaling any parallel application, demonstrates that what previously worked well at large scales does not necessarily extend an additional order or two of magnitude. For example, we have shown that while space-efficient data structures are sufficient for terascale tools, petascale tools must use hierarchically distributed data structures.

Figure 2 shows the merge time results from BG/L with the bit vector optimization in comparison to the original bit vector implementation. The optimized bit vector exhibits logarithmic scaling, in contrast to the original linear scaling, because of the reduction in the data volume being sent through the MRNet network. We achieve this logarithmic scaling despite limitations on the number of communication processes we could launch on BG/L and that we could not employ a fully balanced tree topology. For tools that use a TBON, these results clearly show the importance of designing data structures that are tailored to exploit the logarithmic properties of the network tree effectively. We note that the virtual node mode cases run faster than the co-processor mode cases at equivalent task counts because the merge performance is bound not only by the task count, but also by the number of daemons. Since we are restricted on the number of communication processes we can launch on BG/L, the fanout from the last level of communication processes in coprocessor mode is double that of virtual node mode at equivalent task counts. The optimized bit vector does incur an additional cost in the remapping step, which rearranges the bit vectors into MPI rank order. At $208 \mathrm{~K}$ tasks, this step took only 0.66 seconds. 


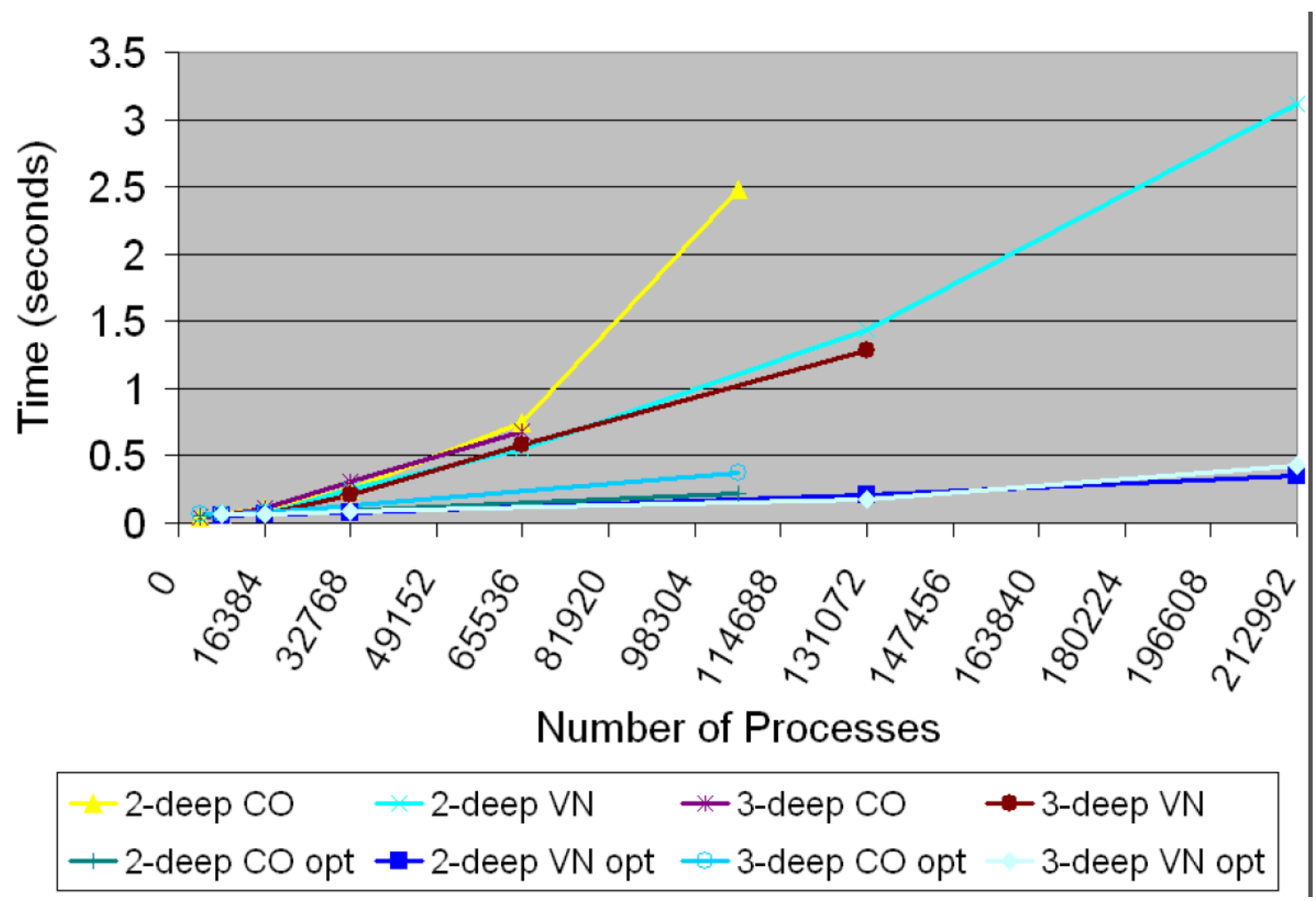

Figure 2: STAT Stack Trace Merge Time Results at Scale (up to 208K Application Processes)

\subsubsection{TAU over MRNet}

The TAU over MRNet (ToM) [1,5] performance monitor integrates TAU performance profiling tool from the University of Oregon with the MRNet scalable infrastructure for runtime program interaction. We built a scalable parallel monitor (based on the ToM prototype) and evaluated its function and performance. We started by defining an abstract monitoring interface to support infrastructure interoperability and leverage MRNet's programming capabilities for analysis filter development. ToM instantiates a tree-based transport network at the start of program execution. Once in place, ToM can be used in a variety of ways for performance data analysis.

Ideally, we want to retrieve and store as much performance detail as the measurement system can provide. However the perturbation caused to the measured application and the transport and storage costs associated with the performance data, require that we trade-off measurement data granularity (in terms of events, time intervals and application ranks) against the costs. One method to vary the level of performance detail is through performance data reduction as the data flows through the transport. This is feasible by distributing performance analyses traditionally performed at the front-end, out to the intermediate transport nodes. ToM implements three such filtering schemes using MRNet, each building upon and extending the previous one: a statistical filter, a histogram filter, and a function classification filter.

Our experiences with the ToM prototype confirm the high return on investment of additional system resources in support of performance monitoring. For instance, with a fanout of 64, overhead 
for additional transport nodes is just over $1.5 \%$ - a reasonable price to pay for the performance benefits. In addition to providing a scalable tree-structured network for consolidated data transfer, the ability to program MRNet for data analysis and reduction relieves the burden on front end processing.

\subsubsection{Fault Tolerant MRNet}

Many high-performance tools, applications and infrastructures, such as Paradyn, STAT, TAU, Ganglia, SuperMon, Astrolabe, Borealis, and MRNet, use data aggregation to synthesize large data sets and reduce data volumes while retaining relevant information content. Hierarchical or treebased overlay networks (TBONs) are often used to execute data aggregation operations in a scalable, piecewise fashion. We developed state compensation, a scalable failure recovery model for high bandwidth, low-latency TBON computations. By leveraging inherently redundant state information found in many TBON computations, state compensation avoids explicit state replication (for example, process checkpoints and message logging) and incurs no overhead in the absence of failures. Further, when failures do occur, state compensation uses a weak data consistency model and localized protocols that allow processes to recover from failures independently and responsively. Based on a formal specification of our data aggregation model, we validated state compensation and identified its assumptions and limitations: state compensation requires that data aggregation operations be associative, commutative and idempotent. We implemented a prototype implementation integrated into the MRNet TBON infrastructure. Our experiments with this framework suggest that for TBONs supporting up to millions of application processes, state compensation can yield millisecond failure recovery.

This research is forming the foundation for ongoing research into building fault tolerant applications, middleware, and services on extreme scale systems.

\subsubsection{Launch Tools at Extreme Scale}

Runtime tools, such as debuggers and performance profilers, are essential to high performance computing. However, several obstacles limit the availability of such tools to one or few platforms and slow the development of new tools. The TDP project (done at Wisconsin ten years ago) noted that the myriad scheduler, job launch, and process control interfaces add severe costs to porting tools to high-end computing environments. On such systems, operating system services and the resource manager (RM) play a critical role in the launching of daemons and their interactions with system resources. Thus, we must port tools to local RMs for efficient execution or even just correct functionality. The variety of RMs, including Condor, LoadLeveler, LSF, SLURM, and YOD, exacerbate this effort. Without a common infrastructure for tool daemon launching, the task of porting $m$ tools to $n$ environments becomes an $m \times n$ effort. Tool developers must implement this capability for each tool/environment combination, often leading to difficult to maintain, ad hoc implementations.

TDP addressed portability but not the issue of scale. With the trend toward systems with $10^{6}$ processors or more, efficient daemon launching is essential. Overheads that previously were merely discouraging are becoming prohibitive. As system scales increase, initialization activities can become bottlenecks that prevent user adoption, particularly for interactive tools like debuggers. Often, such tools must locate their daemons on the same compute nodes as the target application's processes. Further, tools for extreme scale jobs often leverage scalable communication infrastruc- 


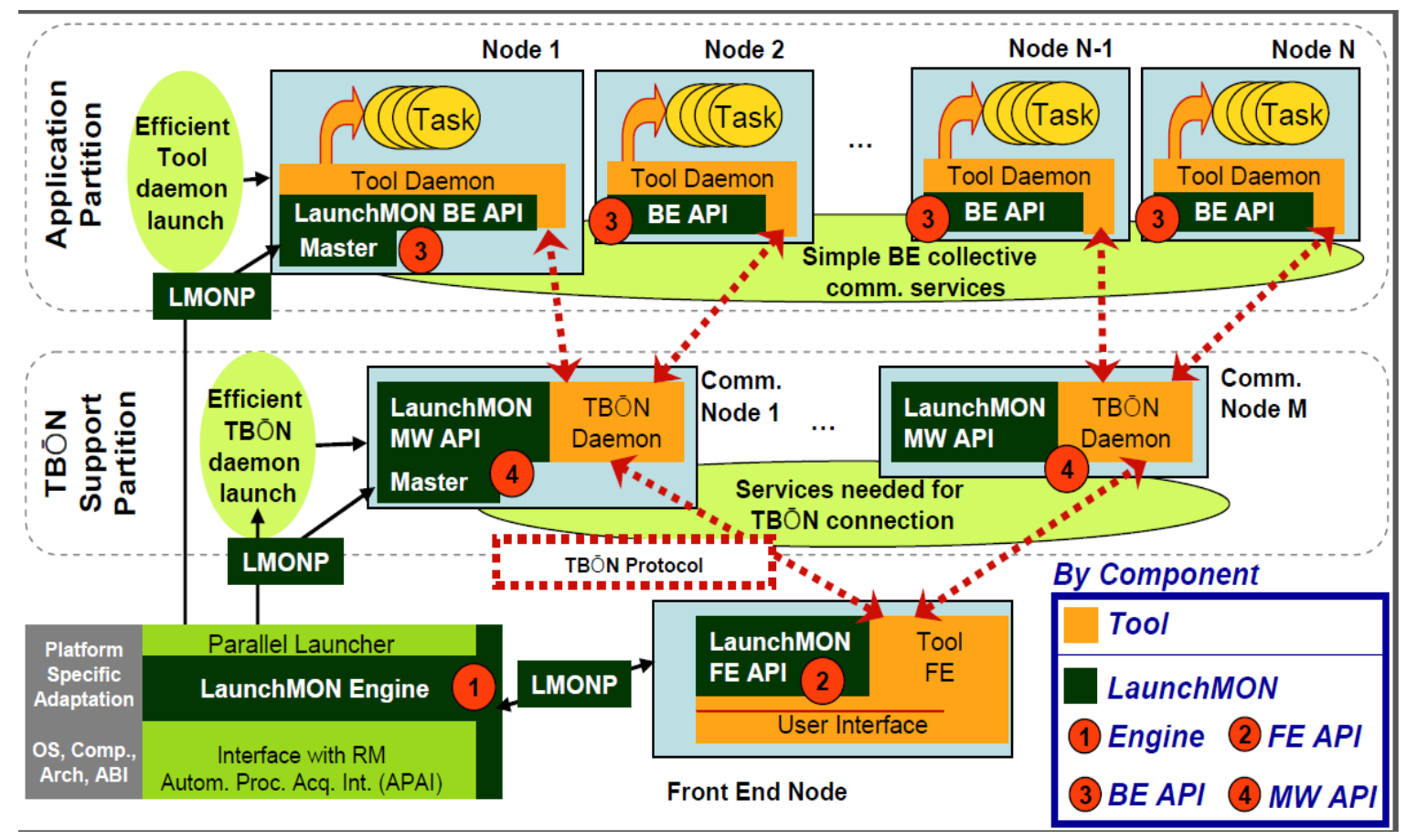

Figure 3: Architecture of LaunchMon

tures that require communication daemons on additional compute resources beyond the target program's allocation.

We seek to help tool developers create highly portable and scalable tools through a standard framework that leverages native system services for tool daemon launching. We developed the key abstractions and mechanisms for a scalable and portable job (and tool) launch facility, and then designed and implemented LaunchMON, a system that embodies these abstractions and mechanisms. LaunchMON can be viewed as a partial TDP implementation that abstracts native RM interfaces and services. Using LaunchMON, tools can automatically run on existing (or future) HPC systems that implement the widely available services upon which we base LaunchMON.

This research made five primary contributions. First, we developed the essential abstractions for a scalable launch facility. Such abstractions are useful to tool developers and compatible with the variety of facilities on existing systems, which we cannot assume will change to accommodate tool developers. Thus, LaunchMON provides the mapping to a uniform interface. Second, we developed a design and implementation for cluster and supercomputer environments that embodies these abstractions. Third, in several case studies we showed how LaunchMON supports existing tools and the creation of new ones. Fourth, one of our new tools was in itself a contribution: Jobsnap provides the first portable and scalable mechanism for users to gather information for each MPI task normally captured through the /proc interface. Last, our experiments and LaunchMON performance model demonstrated that we achieved the goals of portable and scalable tool daemon launching, with overheads an order of magnitude less than existing ad hoc solutions. 


\subsection{Binary Code Analysis, Instrumentation, and Control}

Our project has a long history of accomplishments of tools that operate on binary code, having helped to found the field in the early 1990's. We have consistently released new versions of our Dyninst tool kits, which embody the latest algorithms, techniques, and implementation improvements. As of the end of this grant, we released the DyninstAPI version 7.0 and its related tool kits.

We also continue our exploratory efforts pushing this technology to further limits. Under this funding, we developed new techniques at the foundation of binary code analysis, working precise analysis of binary code, and on the origins of a program's tool chain and authorship from only the binary code. We also developed new path profiling techniques based our binary tool foundation.

\subsubsection{The DyninstAPI Tool Kit Version 7.0}

We summarize the features from this release of Dyninst and its component tool kits. One the key attributes of these tool kits is that they provide an abstract, machine independent interface to binary level code analysis, instrumentation, and control. This feature allows programmer to develop cross-platform tools and do fine-grained operations without needing to understand the lowest level details of a system.

DyninstAPI 7.0: This is the parent library, providing functionality for in-depth analysis of the binary (including control- and data-flow analysis); instrumentation and modification of the binary; and control and monitoring functionality. This release introduced binary rewriting for statically linked binaries on 32- and 64-bit x86 and PowerPC architectures, plus support for the IBM BlueGene/P platform. In addition, significant improvements were made in both parsing and instrumentation speeds.

SymtabAPI 7.0: This library provides a platform independent interface to reading symbol and debug information across a variety of platforms and architectures. In addition, it allows updates to the binary's header and symbol information to support binary rewriting. This update functionality is quite complex, because the standard binary code formats (ELF, XCOFF, and Windows $\mathrm{PE}$ ) were not designed to be updated, so can be quite fragile.

Improvements in 7.0 include support for rewriting dynamic binaries on PowerPC/32, PowerPC/64 and BlueGene/P platforms, support for rewriting statically linked binaries on x86 and x86_64 platforms, and speed and space optimizations.

InstructionAPI 7.0: This library provides a platform independent interface to the representation of an individual instruction, across a variety of processor architectures. It includes an abstract representation of the instructions operation and dataflow information about its operands.

Improvements in 7.0 include support for the PowerPC platform, interface changes for multiple-architecture support, and full integration with the DyninstAPI.

ParseAPI 7.0: This library performs the control-flow parsing of a binary program. It includes the production of a control flow graph (CFG), identifying functions, loops, basic blocks, and instructions in the code. In addition, it labels key points in these objects, such a function entry and exit, loop entry, exit, and body. The CFG provides the basis for support dataflow analysis. Improvements in 7.0 include parsing of executable formats supported by the SymtabAPI debugging and rewriting library (Linux/ELF, Windows PE, AIX/XCOFF), exporting of control flow graph representation (functions, blocks, edges), intraprocedural CFG edges (call and 
return), disassembly of parsed binaries using the InstructionAPI, support for speculative parsing of binaries without debugging symbols, constructed on top of InstructionAPI and SymtabAPI, and full integration with Dyninst.

StackwalkerAPI 2.0: This library provides a platform independent interface to walking the runtime stack, either from a remote process (third party) or from within the running program (first party). It can walk stacks at anytime during execution, including during stack frame set-up and tear-down, and can handle optimized binaries with no stack frames or no function returns (tail call optimizations). In addition, it can understand specialized frames such as those that come from signal handlers and instrumentation code.

Improvements in 2.0 include support for PowerPC/64 platform and significant reduction in memory usage.

ProcControlAPI 1.0: This library provides a platform independent interface to process control and monitoring. It supposes operations such as creating a new process or attaching to a running process; starting, stopping, and stepping a process; reading and writing its address space or registers; and monitoring process creation and termination, program and library loading, breakpoints, and exception occurrences.

This is the first major release of the ProcControlAPI.

DynC 1.0: This library allows instrumentation code snippets to be expressed in C-like language, and is built on top of Dyninst's abstract syntax tree (AST) objects. It includes variable types that distinguished between objects defined in the application program's symbols (including local variables and parameters), instrumentation variables, and temporaries. DynC allows for more rapid prototyping of Dyninst-based programs.

This is the first major release of DynC.

\subsubsection{Provenance of Binary Code: Function Entry Points}

Binary code analysis is a foundational technique in the areas of computer security, performance modeling, and program instrumentation that enables malicious code detection, formal verification, and identification of performance bottlenecks. A binary program contains both code and non-code bytes. The very first step in binary code analysis is to precisely locate all the function entry points (FEPs), which in turn lead to all code bytes. When full symbol or debug information is available this is a trivial step, because the FEPs are explicitly listed. However, malicious programs, commercial software, operating system distributions, and legacy codes all commonly lack symbol information. For these stripped binaries a standard technique is recursive disassembly parsing, which follows program control flow (branches and calls) and finds all functions reachable from the main program entry point. However, this technique cannot statically resolve indirect (pointer based) control flow transfers. Indirect control flow is surprisingly common: on a large set of binaries on our department server, approximately $40 \%$ of functions were unrecoverable through recursive disassembly. These functions lie in gaps between statically discovered functions. To complicate matter, these gaps also contain jump tables, numeric and string constants, padding bytes (both fixed-value and random) and so on. Identifying FEPs within gaps in stripped binaries is of ultimate importance to binary code analysis, but is an unsolved problem.

In this research [12], we developed a novel application of machine learning to large-scale FEP identification. Our main contributions are formulation of FEP identification as a structured classi- 
fication problem, and an implementation that performs significantly better than existing tools at recovering code from stripped binaries. The scale of our experiments, both in terms of the number of binaries in our data sets, and the size of the binaries (which determines the cost of training and inference), far exceeded that of previous work.

Existing techniques have used recursive disassembly parsing and heuristics to locate code in stripped binaries (Theiling 2000; Cifuentes \& Emmerik 2000). Several tools, including Dyninst (Hollingsworth, Miller, \& Cargille 1994) and IdaPro (Data Rescue 2007), use a small number of instruction patterns to identify FEPs in gaps. These patterns are created manually for particular compilers. For example, Dyninst searches binaries on the Intel IA-32 platform for a common function preamble pattern that sets up a stack frame: (push ebp | mov esp, ebp). Other efforts have incorporated simple unigram and bigram instruction models to augment pattern heuristics for locating code in stripped binaries (Kruegel et al. 2004). These heuristics and simple statistical methods cannot adapt to variations in compiler, optimization level, and post-compilation optimization tools, all of which significantly perturb or even optimize away expected instruction sequences at FEPs.

We overcame these challenges by incorporating both content and structure features in identifying FEPs. Our content features consists of idioms, instruction patterns around FEPs. Importantly, we perform automatic feature selection from large corpora of binaries, which gives us flexibility and coverage not possible in hand-coded patterns. Our structure features follow from two properties of binary code:

1. An instruction at byte-offset $\mathrm{x}$ within the binary can span several bytes. If so, the locations $x$ and $x+1$ represent conflicting (overlapping) parses, and are unlikely to both be FEPs;

2. The disassembly starting from $\mathrm{x}$ can contain a call instruction that calls offset $x_{0}$. If we believe that $x$ is an FEP, then $x_{0}$ probably is too.

By using both content and structure features, we were able to identify FEPs in gaps with significantly higher accuracy than existing techniques.

We evaluated our models on several large real-world data sets of Linux and Windows binaries and, compared against the best existing tools (for example, IDAPro), we achieved substantial improvements in the ability to correctly identify FEPs while also reducing false positives.

\subsubsection{Provenance of Binary Code: Tool Chain Provenance}

Program binaries are often the subject of analysis in the areas of computer security, performance modeling, and program instrumentation and debugging. Security analysts and antivirus vendors are confronted daily with malicious programs whose behavior they must analyze and understand, and whose origins and relationships to existing threats are of paramount importance. Developers of debugging, performance analysis, and instrumentation software must design tools useful in the face of myriad variations of source languages, programming idioms, and diverse compiler families and types of optimizations. The question of binary program provenance - of the characteristics of a program that derive from its path from source code to executable form-informs many aspects of binary analysis. The utility of program provenance can be straightforward, as in the case of software forensics where authorship and code similarity are key details. In other domains it can be more subtly useful, aiding the development of binary tools and analysis capabilities targeted at specific compilers or source languages. When presented with only with a program binary or a snippet 
of binary code, details of program provenance are not readily apparent. The black box between the program author and the program binary affords little foothold for tailored tools or analyses.

We developed a novel method for identifying the source compiler of program binaries, a major element of program provenance [3]. We formulated compiler identification as a structured learning task, automatically building models that classify sequences of binary code by the generating compiler. Because our approach relies only on characteristics of the binary code and not on metadata or other details of program headers, it is applicable even when such information has been stripped or is otherwise unavailable. Furthermore, because our method classifies sequences of code instead of whole binaries, it can be used even when codes produced by multiple compilers coexist within a program binary, such as statically linked library code. Our tool extracts compiler provenance with high accuracy even in such complex programs.

Our previous research into precise static parsing of stripped program binaries-a foundational technique for binary code analysis - used compiler-specific models of code at function entry points to extend traditional parsing techniques that perform poorly without debugging symbols. The existing approach requires prior knowledge of the source compiler, which may not be available. We augmented the binary code parser to relax the known-compiler requirement, and show that adding inferred source compiler labels improves the precision of analysis for binaries of unknown provenance. While the high accuracy of the existing parser allows only small improvement in absolute terms, adding compiler provenance reduces parsing errors by $18 \%$.

\subsubsection{Incremental Path Profiling}

Profiling is a key component of the optimization process, and is vital to achieving high levels of performance. Function-level profiling, however, is limited in that it only gathers information about the aggregate behavior of functions in a program. This information is not sufficient to completely characterize a function whose behavior varies between calls; for example, a function that performs well with one type of input and poorly with a second. Call-path profiling, an extension to function-level profiling, is a mechanism by which the information gathered by the profiler is attributed to paths through the call graph instead of being aggregated together. This more complete profiling information is particularly useful when optimizing programs that use libraries, such as those for communication (MPI or PVM), linear algebra (ScaLAPACK), or threading.

Call-path profiling allows a user to identify and characterize performance problems that depend on from where a function is called, rather than on the function itself. A function may complete quickly for most invocations, but require excessive time when executed along a particular callchain. For example, a communication function may block waiting for input in particular cases, or a utility function may perform poorly for a particular input. Call-path profiling also clarifies performance problems caused by inefficient calling behavior, such as when a function is called an excessive number of times. For example, a math function may be called multiple times with identical input. Function-level profiling would mis-identify the math function as a bottleneck, but callpath profiling would identify the calling behavior as the problem.

Previous instrumenting call-path profilers have traced all functions in the program. These tools have tracked the call-path at all times by instrumenting all function entries and exits. While this complete information can be useful in some cases, it generates substantial overhead and can slow the program by up to $700 \%$. Other profilers have used sampling instead of instrumentation to lessen the overhead involved in profiling, but cannot generate precise results. Our work [10] is 
motivated by the insight that users often do not desire information for every function in the program, focusing instead on a particular set of functions for which they need more exact information about their behavior. In this case, the information offered by a whole-program profiler is both unnecessarily broad and too expensive. By focusing instead on particular functions, a profiler may reduce the overhead involved in call-path profiling. However, previous call-path profiling algorithms are not amenable to this partial profiling approach, as they require whole-program instrumentation to track the call-path. We developed a partial profiling method that does not require whole-program instrumentation to generate accurate call-path profiling results. Our technique complements other profiling tools and approaches, and provides an efficient method for profiling the call path of particular functions.

In this research, we developed a technique that we call incremental call-path profiling. Incremental call-path profiling allows a user to profile particular functions rather than the entire program. The profile data may be examined while the program is running, and the user may refine both the selected functions and the metrics gathered while the program is still running. By profiling particular functions, we provide the benefits of call-path profiling while significantly reducing the overhead incurred.

This method is distinguished by five key capabilities:

- Targeted: Our technique is able to profile particular functions, and only these functions are instrumented;

- Dynamic: Profiling instrumentation can be inserted and removed at run-time, without prior program preparation;

- Run-Time Results: Up-to-date profiling results are available as the program runs, without requiring a postprocessing phase;

- Cost-effective: Overhead is incurred only when the profiled functions are executed, allowing the use of more complex metrics;

- Complete: The entire call-path is captured, including dynamic calls through function pointers.

This research developed a method for incremental call-path profiling. Our key technique is the use of a lightweight stack walk to determine the complete call-path. This method allows us to determine the complete call-chain without requiring whole-program instrumentation. We implemented our techniques in iPath, a prototype incremental call-path profiler. This tool is capable of gathering a wide variety of performance metrics for the call-path while instrumenting only the functions of interest to the user. iPath supports both counter and timer-based performance metrics and is capable of calculating metrics based on both hardware counters.

As a test of our technique, we applied iPath to two real systems, the su3_rmd distributed quantum chromodynamics simulation built on the MILC framework, and the instrumentation daemon of the Paradyn dynamic profiler. In both applications, we found and removed significant call-path specific bottlenecks. Our modifications resulted in dramatic improvements, a $45 \%$ decrease in running time of su3_rmd, and an almost 98\% decrease in Paradyn instrumentation time. 


\section{Publications}

[1] Aroon Nataraj, Allen D. Malony, Alan Morris, Dorian C. Arnold, and Barton P. Miller, "A Framework for Scalable Parallel Performance Monitoring", Concurrency and Computation: Practice and Experience 22, 6, June 2010, pp. 720-735.

[2] Dorian C. Arnold and Barton P. Miller, "Scalable Failure Recovery for High-performance Data Aggregation", International Parallel and Distributed Processing Symposium (IPDPS), Atlanta, April 2010.

[3] Nathan E. Rosenblum, Barton P. Miller and Xiaojin (Jerry) Zhu, "Extracting Compiler Provenance from Program Binaries", $9^{\text {th }}$ ACM SIGNPLAN-SIGSOFT Workshop on Program Analysis for Software Tools and Engineering (PASTE), Toronto, June 2010.

[4] Greg L. Lee, Dong H. Ahn, Dorian C. Arnold, Bronis R. de Supinski, Barton P. Miller, Martin Schulz, and Benjamin Liblit, "Lessons learned at 208K: Towards Debugging Millions of Cores", Supercomputing 2008 (SC2008), Austin, TX, November 2008.

[5] Aroon Nataraj, Allen D. Malony, Alan Morris Dorian C. Arnold, and Barton P. Miller, "In Search of Sweet-Spots in Parallel Performance Monitoring", 2008 IEEE International Conf. on Cluster Computing (Cluster 2008), Tsukuba, Japan, September 2008.

[6] Dong H. Ahn, Dorian C. Arnold, Bronis R. de Supinski, Greg L. Lee, Barton P. Miller, and Martin Schulz, "Overcoming Scalability Challenges for Tool Daemon Launching", $37^{\text {th }}$ International Conference on Parallel Processing (ICPP-08), Portland, OR, September 2008.

[7] Greg L. Lee, Dong H. Ahn, Dorian C. Arnold, Bronis R. de Supinski, Barton P. Miller, and Martin Schulz, "Benchmarking the Stack Trace Analysis Tool for BlueGene/L", Parallel Computing 2007 (ParCo), Minisymposium on Scalability and Usability of HPC Programming Tools, Jülich, Germany, September 2007.

[8] Dorian C. Arnold, Gary D. Pack and Barton P. Miller, "Tree-based Overlay Networks for Scalable Applications", $11^{\text {th }}$ International Workshop on High-Level Parallel Programming Models and Supportive Environments (HIPS 2006), Rhodes, Greece, April 2006.

[9] Dorian C. Arnold, Dong H. Ahn, Bronis R. de Supinski, Greg Lee, Barton P. Miller, and Martin Schulz, "Stack Trace Analysis for Large Scale Debugging", $21^{\text {st }}$ IEEE International Parallel and Distributed Processing Symposium (IPDPS), Long Beach, California, March 2007.

[10] Andrew R. Bernat and Barton P. Miller, "Incremental Call-Path Profiling", Concurrency: Practice and Experience 19, 11, August 2007, pp. 1533-1547.

[11] Swetha Krishnan, Giridhar Ravipati, Andrea Arpaci-Dusseau, Remzi Arpaci-Dusseau, Barton P. Miller, "Fingerprinting NFS Failure Policy", $3^{\text {rd }}$ International Workshop on Storage Security and Survivability (StorageSS 2007), Alexandria, VA, October 2007.

[12] Nathan E. Rosenblum, Xiaojin (Jerry) Zhu, Barton P. Miller and Karen Hunt, "Learning to Analyze Binary Computer Code", $23^{\text {rd }}$ AAAI Conference on Artificial Intelligence (AAAI 2008), Chicago, July 2008.

[13] Nathan Rosenblum, Gregory Cooksey, and Barton P. Miller, "Virtual Machine-Based Circumvention of Self-Checksumming Code", International Conference on Virtual Execution Environments (VEE 2008), March 2008, Seattle. 


\section{Students Supported ANd Student Progress}

A total of nine graduate Research Assistants were supported during the reporting period, with three finishing their Ph.D. degrees. No post doctoral nor undergraduate student were supported during this period.

\begin{tabular}{ll} 
PhD Students & MS Students \\
\hline Dorian Arnold & Todd Frederick \\
Drew Bernat & Laune Harris \\
Nathan Rosenblum & Todd Miller \\
& Gary Pack \\
& Min Qiu \\
& Giridhar Ravipati
\end{tabular}

\section{OUTREACH AND Transitions}

Following is a summary of highlights from our technology transitions:

- Cray Research: Cray adopted MRNet and other related tools as the foundation for their new suite of scalable "Abnormal Process Termination" tools.

- The ORNL team presented MRNet for the Cray XT to the team from the Coordinated Infrastructure for Fault Tolerant Systems (CiFTS) project as a potential "fault tolerant backplane" for their infrastructure.

- Barton Miller was appointed to the Petascale Software Advisory Committee, NSF/“Blue Waters".

- Cray developed at least one tool using the result of our work in bringing the MRNet infrastructure to the Cray XT platform. MRNet is part of the software stack delivered on current Cray systems.

- The STAT tool was used in production runs on the acceptance of the LLNL IBM BG/Q Sequoia machine and was used to diagnose a system problem at over a million processes. This was a problem that IBM was not able to diagnose with their own tools.

- MRNet and Dyninst have been used in a wide variety of tools from the top research groups at University of Oregon, Barcelona Supercomputer Center, Jülich Supercomputer Center, Rice University, Krell Labs, Lawrence Livermore National Lab, and Los Alamos National Lab.

\section{External Presentations}

In addition to conference and workshop papers listed in Section 2 above, the following presentations were given:

- Barton P. Miller, Invited talk, Hebrew University of Jerusalem, "Tree-based Overlay Networks for Scalable Middleware and Systems", December, 2008.

- Barton P. Miller, Invited talk, Forschungszentrum Jülich, "Random Testing with 'Fuzz': 18 Years of Finding Bugs", Germany, October 2008.

- Barton P. Miller, Invited talk, Epic Systems, Inc., "Random Testing with 'Fuzz': 18 Years of Finding Bugs", Madison, WI, March 2009. (Epic Systems is the largest online medical records provider in the country with over 130 million patients online.)

- Barton P. Miller, Autonomous University of Barcelona, "Tree-based Overlay Networks for Building Extreme Scale Tools", October 2009.

- Barton P. Miller, Invited task, University of California, San Diego, "Scalability in Tools and Middleware", La Jolla, CA, October 2010. 
- Barton P. Miller, Keynote Address, "How much Security is Enough? And at What Cost?", Open Grid Forum 28, Munich Germany, March 2010.

\section{AWARDS AND HONORS}

- Barton Miller: University of Wisconsin Vilas Associate Award.

- $\quad$ MRNet, as part of the Stack Trace Debugging Tool (STAT) won an R\&D 100 Award in 2011. 